\title{
Comparison of Market Parameters for Jump-Diffusion Distributions Using Multinomial Maximum Likelihood Estimation
}

\author{
Floyd B. Hanson and Zongwu Zhu
}

\begin{abstract}
Previously, we have shown that the proper method for estimating parameters from discrete, binned stock $\log$ returns is the multinomial maximum likelihood estimation, and its performance is superior to the method of least squares. Also, useful formulas have been derived for the density for jump-diffusion distributions. Numerically, the parameter estimation can be a large scale nonlinear optimization, but we have successfully implemented variants of multi-dimension direct search methods. In this paper, three jump-diffusion models using different jump-amplitude distributions are compared. These jump-amplitude distributions are the normal, uniform and double-exponential distribution. The parameters of all three models are fit to the Standard and Poor's 500 logreturn market data, given the same first moment and second central moments. Our main results are first that uniform jump distribution has superior qualitative performance since it produces genuine fat tails that are typical of market data, whereas the other two have exponentially thin tails. Secondly, the uniform distribution is quantitatively better overall as measured by the closeness of both the skewness and kurtosis coefficients to the data, although the double-exponential is best on skewness while worst on kurtosis. However, the lognormal model has a big advantage in computational costs of parameter estimation compared with the others, while the double-exponential is most costly due to having one more model parameter to fit.
\end{abstract}

\section{INTRODUCTION}

Despite the great success of Black-Scholes options model [2], in option pricing, this pure log-normal diffusion model fails to reflect the three empirical phenomena: (1) the large random fluctuations such as crashes or rallies; (2) the nonnormal features, that is, negative skewness and leptokurtic (peakedness) behavior in the stock log-return distribution; (3) the implied volatility smile, that is, the implied volatility is not a constant as in the Black-Scholes model.

Therefore, many different models are proposed to modify the Black-Scholes model so as to represent the above three empirical phenomena. Some models are proposed to incorporate the volatility smile, for example, Andersen, Benzoni and Lund [1] have made elaborate estimations to fit jump-diffusion models with log-normal jump-amplitudes, stochastic volatility and other features. Some models are proposed to incorporate the asymmetric features of the stock log-return distributions. Merton [10] introduced the jumpdiffusion model in financial modeling, using a Poisson process for the jump timing and a log-normal process

The work is supported by the National Science Foundation under Grant DMS-02-07081. The content of this material is that of the authors and does not necessarily reflect the views of the National Science Foundation.

Department of Mathematics, Statistics, and Computer Science, M/C 249, University of Illinois, Chicago, IL 60607-7045 hanson@math.uic.edu and zzhu@math.uic.edu for the jump-amplitudes to describe the market crashes or rallies. Recently, Kou [9] proposed a jump-diffusion model with a log-double-exponential process for the jumpamplitude. Since crashes or rallies are rare events, the Poisson process is reasonable for the timing of jumps. However, there is a problem in choosing the log-normal or log-double-exponential process for the jump-amplitude since the exponentially small tails of the log-normal and logdouble-exponential distributions are contrary to the flat and thick tails of the long time financial market log-return data. Around the near-zero peak of the log-double-exponential and the log-normal, the jumps are small, so are not too different from the continuous diffusion fluctuations. When the jumps are large, then the density tails are exponentially small, but the large jumps of the data are more persistent. Moreover, an infinite jump domain is unrealistic, since the jumps should be bounded in a real world financial markets and an infinite domain leads to unrealistic restrictions in portfolio optimization [5].

So, Hanson and Westman [4] proposed one jumpdiffusion model with log-uniform jump-amplitude. Most recently, Hanson, Westman and Zhu [8] showed that for IID simulations that the binned distribution is multinomial. They estimated the market parameters for this log-uniform model by subsequent multinomial maximum likelihood method to fit financial market distributions such as the Standard and Poor's 500 stock index. The estimation of the kurtosis differed by a very small amount, $+0.78 \%$, from the observed value. However, the estimation of the skewness differed significantly from the observed value, by $-47 \%$. In this paper, the value of the skewness of the log-uniform model is greatly improved using more accurate computations here.

The main purpose of this paper is to compare the performance of three jump-diffusion models whose jumpamplitudes are the log-normally, log-uniformly and logdouble-exponentially distributed. The measures of performance are the skewness, kurtosis and computational costs.

\section{SOME THEORETICAL RESULTS ABOUT THESE JUMP-DIFFUSION MODELS}

\section{A. Stock Return Process, $\mathbf{S}(\mathbf{t})$}

The following stochastic differential equation (SDE) is used to model the dynamics of the asset price, $S(t)$ :

$$
d S(t)=S(t)\left(\mu_{d} d t+\sigma_{d} d W(t)+J(Q) d P(t)\right),
$$

where $\mu_{d}$ is the drift coefficient, $\sigma_{d}$ is the diffusive volatility, $W(t)$ is the stochastic diffusion process, $J(Q)$ is the Poisson 
jump-amplitude, $Q$ is its underlying Poisson amplitude mark process, $P(t)$ is the standard Poisson jump process with joint mean and variance $\mathrm{E}[P(t)]=\lambda t=\operatorname{Var}[P(t)]$.

\section{B. Stock Log-Return Process, $\ln (\mathbf{S}(\mathbf{t}))$}

The stock log-return $\ln (S(t))$ can be transformed to a simpler jump-diffusion stochastic differential equation (SDE) upon use of the stochastic chain rule [7],

$$
d[\ln (S(t))]=\mu_{l d} d t+\sigma_{d} d W(t)+Q d P(t),
$$

where $\mu_{l d} \equiv \mu_{d}-0.5 \sigma_{d}^{2}$ can be called the log-diffusive (ld) drift. For simplicity the log-transformed jump-amplitude is taken as the mark,

$$
Q=\ln (J(Q)+1)
$$

\section{Log-Normal Jump Distribution}

Let the density of the jump-amplitude mark $Q$ be normal

$$
\phi_{Q}(q)=\phi^{(n)}\left(q ; \mu_{j}, \sigma_{j}^{2}\right)
$$

where $\phi^{(n)}\left(q ; \mu_{j}, \sigma_{j}^{2}\right)$ is the normal density with mean $\mu_{j}$ and variance $\sigma_{j}^{2}$. The log-normal jump-amplitude jumpdiffusion model was used in [10], [1], [3] and others.

For the density for this jump-diffusion model with lognormal jump-amplitude, Hanson and Westman [3] proved the following theorem:

Theorem: The probability density for the linear jumpdiffusion log-return increment $\Delta[\ln (S(t))]$ with log-normal jump-amplitude is given by

$$
\begin{aligned}
\phi^{(\mathrm{jd})}(x)= & \sum_{k=0}^{\infty} p_{k}(\lambda \Delta t) \\
& \cdot \phi^{(n)}\left(x ; \mu_{l d} \Delta t+k \mu_{j}, \sigma_{d}^{2} \Delta t+k^{2} \sigma_{j}^{2}\right),
\end{aligned}
$$

for $-\infty<x<+\infty$, where $p_{k}(\Lambda)=e^{-\Lambda} \Lambda^{k} / k$ ! is the Poisson distribution with parameter $\Lambda$ and $k$ jumps, where $\Delta \mathbf{t}$ is the corresponding trading time increment.

This theorem is based upon the law of total probability [7] resulting in the sum over all $k$ Poisson jumps, the convolution theorem [7] yielding the density of the logjump-diffusion conditioned on there being $k$ jumps, and the fact that the convolution of two normals is also normal [7]. Given $k$, the density of the jump term is simply $\phi_{k Q}(q)=\phi_{Q}(q / k) / k$. The theorem is posed as the logreturn increment rather than for the infinitesimal, because the time between trading data is small but not infinitesimal. For the purpose of comparison, we use more terms of the expansion than we have in our other papers to provide more accurate estimations since we are dealing with small but not very small time steps and the scale these time steps can be magnified by a jump rate that includes many small jumps that are indistinguishable from the fluctuations of the diffusion process.
1) Basic Moments of Log-Return Increments $\Delta[\ln (\mathbf{S}(\mathbf{t}))]$ for Log-Normal Jumps::

- 1st moment:

$$
M_{1}^{(\mathrm{jd})} \equiv \mathrm{E}[\Delta[\ln (S(t))]]=\mu_{l d} \Delta t+\mu_{j} \lambda \Delta t .
$$

- 2nd moment:

$$
\begin{aligned}
M_{2}^{(\mathrm{jd})} & \equiv \operatorname{Var}[\Delta[\ln (S(t))]] \\
& =\sigma_{d}^{2} \Delta t+\left(\sigma_{j}^{2}(1+\lambda \Delta t)+\mu_{j}^{2}\right) \lambda \Delta t .
\end{aligned}
$$

- 3rd moment:

$$
\begin{aligned}
M_{3}^{(\mathrm{jd})} & \equiv \mathrm{E}\left[\left(\Delta[\ln (S(t))]-M_{1}^{(j d)}\right)^{3}\right] \\
& =\left(3 \mu_{j} \sigma_{j}^{2}+\mu_{j}^{3}\right) \lambda \Delta t+6 \mu_{j} \sigma_{j}^{2}(\lambda \Delta t)^{2} .
\end{aligned}
$$

- 4th moment:

$$
\begin{aligned}
M_{4}^{(\mathrm{jd})} \equiv & \mathrm{E}\left[\left(\Delta[\ln (S(t))]-M_{1}^{(j d)}\right)^{4}\right] \\
= & \left(\mu_{j}^{4}+3 \sigma_{j}^{4}+6 \mu_{j}^{2} \sigma_{j}^{2}\right) \lambda \Delta t \\
& +\left(3 \mu_{j}^{4}+21 \sigma_{j}^{4}+30 \mu_{j}^{2} \sigma_{j}^{2}\right)(\lambda \Delta t)^{2} \\
& +6 \sigma_{d}^{2} \Delta t\left(\sigma_{j}^{2}+\mu_{j}^{2}\right) \lambda \Delta t \\
& +3\left(\sigma_{d} \Delta t\right)^{2}+\left(6 \mu_{j}^{2} \sigma_{j}^{2}+18 \sigma_{j}^{4}\right)(\lambda \Delta t)^{3} \\
& +6 \sigma_{d}^{2} \Delta t \sigma_{j}^{2}(\lambda \Delta t)^{2}+3 \sigma_{j}^{4}(\lambda \Delta t)^{4} .
\end{aligned}
$$

\section{Log-Uniform Jump Distribution}

Let the density of the jump-amplitude mark $Q$ be uniform

$\phi_{Q}(q)=\left(H\left(Q_{b}-q\right)-H\left(Q_{a}-q\right)\right) /\left(Q_{b}-Q_{a}\right)$,

where $Q_{a}<0<Q_{b}$ and $H(x)$ is the Heaviside unit step function. The mark $Q$ has moments, $\mu_{j} \equiv \mathrm{E}_{Q}[Q]=$ $0.5\left(Q_{b}+Q_{a}\right), \sigma_{j}^{2} \equiv \operatorname{Var}_{Q}[Q]=\left(Q_{b}-Q_{a}\right)^{2} / 12$. The original jump-amplitude $J$ has mean $\mathrm{E}[J(Q)]=\left(\exp \left(Q_{b}\right)-\right.$ $\left.\exp \left(Q_{a}\right)\right) /\left(Q_{b}-Q_{a}\right)-1$ and $\log$-uniform distribution

$$
\Phi_{J}(x)=\ln \left((x+1) /\left(J_{a}+1\right)\right) / \ln \left(\left(J_{b}+1\right) /\left(J_{a}+1\right)\right)
$$

on $\left[J_{a}, J_{b}\right]$, where $J_{a} \equiv J\left(Q_{a}\right)$ and $J_{b} \equiv J\left(Q_{b}\right)$.

For the density of the jump-diffusion model with loguniform jump-amplitude, the following theorem is given in [4].

Theorem: The probability density for the linear jumpdiffusion, log-return increment $\Delta[\ln (S(t))]$ with $\log$ uniform jump-amplitude is given by

$$
\begin{aligned}
\phi^{(\mathrm{jd})}(x)= & p_{0}(\lambda \Delta t) \phi^{(n)}\left(x ; \mu_{l d} \Delta t, \sigma_{d}^{2} \Delta t\right) \\
& +\sum_{k=1}^{\infty} p_{k}(\lambda \Delta t) \\
& \cdot \frac{\Phi^{(n)}\left(x-k Q_{b}, x-k Q_{a} ; \mu_{l d} \Delta t, \sigma_{d}^{2} \Delta t\right)}{k\left(Q_{b}-Q_{a}\right)},
\end{aligned}
$$

for $-\infty<x<+\infty$, where $p_{k}(\Lambda)=e^{-\Lambda} \Lambda^{k} / k$ ! is the Poisson distribution with parameter $\Lambda$ and $k$ jumps and $\Phi^{(n)}\left(x_{1}, x_{2} ; \mu, \sigma^{2}\right)$ is the normal distribution in interval $\left[x_{1}, x_{2}\right]$, where $\Delta \mathbf{t}$ is the corresponding trading time increment.

The justification of this theorem is similar to that as for the log-normal, except that the $k$-jump conditioned 
convolution leads to a combined jump-diffusion normaluniform density given in (II.6) that we call the secantnormal density since the density is the secant of the normal distribution.

1) Basic Moments of Log-Return Increments $\Delta[\ln (\mathbf{S}(\mathbf{t}))]$ for Log-Uniform Jumps::

- 1st moment:

$$
M_{1}^{(\mathrm{jd})} \equiv \mathrm{E}[\Delta[\ln (S(t))]]=\mu_{l d} \Delta t+\mu_{j} \lambda \Delta t .
$$

- 2nd moment:

$$
\begin{aligned}
M_{2}^{(\mathrm{jd})} & \equiv \operatorname{Var}[\Delta[\ln (S(t))]] \\
& =\sigma_{d}^{2} \Delta t+\left(\sigma_{j}^{2}(1+\lambda \Delta t)+\mu_{j}^{2}\right) \lambda \Delta t .
\end{aligned}
$$

- 3rd moment:

$$
\begin{aligned}
M_{3}^{(\mathrm{jd})} & \equiv \mathrm{E}\left[\left(\Delta[\ln (S(t))]-M_{1}^{(j d)}\right)^{3}\right] \\
& =\left(3 \mu_{j} \sigma_{j}^{2}+\mu_{j}^{3}\right) \lambda \Delta t+6 \mu_{j} \sigma_{j}^{2}(\lambda \Delta t)^{2} .
\end{aligned}
$$

- 4th moment:

$$
\begin{aligned}
M_{4}^{(\mathrm{jd})} \equiv & \mathrm{E}\left[\left(\Delta[\ln (S(t))]-M_{1}^{(j d)}\right)^{4}\right] \\
= & \left(\mu_{j}^{4}+1.8 \sigma_{j}^{4}+6 \mu_{j}^{2} \sigma_{j}^{2}\right) \lambda \Delta t \\
& +\left(3 \mu_{j}^{4}+12.6 \sigma_{j}^{4}+30 \mu_{j}^{2} \sigma_{j}^{2}\right)(\lambda \Delta t)^{2} \\
& +6 \sigma_{d}^{2} \Delta t\left(\sigma_{j}^{2}+\mu_{j}^{2}\right) \lambda \Delta t \\
& +3\left(\sigma_{d}^{2} \Delta t\right)^{2}+\left(6 \mu_{j}^{2} \sigma_{j}^{2}+10.8 \sigma_{j}^{4}\right)(\lambda \Delta t)^{3} \\
& +6 \sigma_{d}^{2} \Delta t\left(\sigma_{j}^{2}+\mu_{j}^{2}\right)(\lambda \Delta t)^{2} \\
& +1.8 \sigma_{j}^{4}(\lambda \Delta t)^{4} .
\end{aligned}
$$

Note that the formulas for the first three moments are the same for both log-normal and log-uniform jumps.

\section{E. Log-Double-Exponential Jump Distribution}

Let the density of the jump-amplitude mark $Q$ be doubleexponential

$$
\phi_{Q}(q)=\frac{p}{\mu_{1}} e^{\frac{q}{\mu_{1}}} I_{\{q<0\}}+\frac{(1-p)}{\mu_{2}} e^{\frac{-q}{\mu_{2}}} I_{\{q \geq 0\}},
$$

where $\mu_{1}>0$ and $\mu_{2}>0$ are one-sided means, and $0<$ $p<1$ represents the probability of downward jumps while $1-p$ is the probability of upward jumps. The set indicator function is $I_{\{\mathcal{S}\}}$ for set $\mathcal{S}$. The mark $Q$ has moments, $\mu_{j} \equiv$ $\mathrm{E}_{Q}[Q]=-p \mu_{1}+(1-p) \mu_{2}, \sigma_{j}^{2} \equiv \operatorname{Var}_{Q}[Q]=p(2-p) \mu_{1}^{2}+$ $2 p(1-p) \mu_{1} \mu_{2}+\left(1-p^{2}\right) \mu_{2}^{2}$.

Similar to the theorem in [3], we get the following theorem:

Theorem: The probability density for the linear jumpdiffusion log-return increment $\Delta[\ln (S(t))]$ with log-double- exponential jump-amplitude is given by

$$
\begin{aligned}
\phi^{(\mathrm{jd})}(x)= & p_{0}(\lambda \Delta t) \phi^{(n)}\left(x ; \mu_{l d} \Delta t, \sigma_{d}^{2} \Delta t\right) \\
& +\sum_{k=1}^{\infty} \frac{p_{k}(\lambda \Delta t)}{k} \\
& \cdot\left(\frac{p}{\mu_{1}} \exp \left(\frac{x-\mu_{l d} \Delta t+0.5 \sigma_{d}^{2} \Delta t /\left(k \mu_{1}\right)}{k \mu_{1}}\right)\right. \\
& \cdot \Phi^{(n)}\left(-x ; \bar{\mu}_{1}, \sigma_{d}^{2} \Delta t\right) \\
& +\frac{1-p}{\mu_{2}} \exp \left(\frac{\mu_{l d} \Delta t-x+0.5 \sigma_{d}^{2} \Delta t /\left(k \mu_{2}\right)}{k \mu_{2}}\right) \\
& \left.\cdot\left(1-\Phi^{(n)}\left(-x ; \bar{\mu}_{2}, \sigma_{d}^{2} \Delta t\right)\right)\right),
\end{aligned}
$$

for $-\infty<x<+\infty$, where $\bar{\mu}_{1} \equiv \sigma_{d}^{2} \Delta t /\left(k \mu_{1}\right)-\mu_{l d} \Delta t$, $\bar{\mu}_{2} \equiv-\sigma_{d}^{2} \Delta t /\left(k \mu_{2}\right)-\mu_{l d} \Delta t$, and $\Delta \mathbf{t}$ is the corresponding trading time increment.

1) Basic Moments of Log-Return Increments $\Delta[\ln (\mathbf{S}(\mathbf{t}))]$ for Log-Double-exponential Jumps::

- 1st moment:

$$
\begin{aligned}
M_{1}^{(\mathrm{jd})} & \equiv \mathrm{E}[\Delta[\ln (S(t))]] \\
& =\mu_{l d} \Delta t+\left(-p \mu_{1}+(1-p) \mu_{2}\right) \lambda \Delta t .
\end{aligned}
$$

- 2nd moment:

$$
\begin{aligned}
M_{2}^{(\mathrm{jd})} \equiv & \operatorname{Var}[\Delta[\ln (S(t))]] \\
= & \sigma_{d}^{2} \Delta t+2\left(p\left(\mu_{1}^{2}-\mu_{2}^{2}\right)+\mu_{2}^{2}\right) \lambda \Delta t \\
& +\left(p(2-p) \mu_{1}^{2}+2 p(1-p) \mu_{1} \mu_{2}\right. \\
& \left.+\left(1-p^{2}\right) \mu_{2}^{2}\right)(\lambda \Delta t)^{2} .
\end{aligned}
$$

- 3rd moment:

$$
\begin{aligned}
M_{3}^{(\mathrm{jd})} \equiv & \mathrm{E}\left[\left(\Delta[\ln (S(t))]-M_{1}^{(j d)}\right)^{3}\right] \\
= & 2(\lambda \Delta t)^{3}\left(3 p(p-1) \mu_{1}^{3}-p^{3}\left(\mu_{1}^{3}+\mu_{2}^{3}\right)\right. \\
& +3 p^{2} \mu_{1} \mu_{2}^{2}-3 p^{3} \mu_{1} \mu_{2}\left(\mu_{2}+\mu_{1}\right) \\
& \left.+\mu_{2}^{3}+3 p(2 p-1) \mu_{1}^{2} \mu_{2}\right) \\
& +6(\lambda \Delta t)^{2}\left(-p \mu_{1}^{2} \mu_{2}+p \mu_{2}^{2} \mu_{1}-p^{2} \mu_{1} \mu_{2}^{2}\right. \\
& -p^{2} \mu_{2}^{3}-p \mu_{2}^{3}+2 \mu_{2}^{3}+p^{2} \mu_{1}^{3}+p^{2} * \mu_{1}^{2} \mu_{2} \\
& \left.-3 p \mu_{1}^{3}\right)+6 \lambda \Delta t\left((1-p) \mu_{2}^{3}-p \mu_{1}^{3}\right) ;
\end{aligned}
$$

- 4th moment:

$$
\begin{aligned}
M_{4}^{(\mathrm{jd})} \equiv & \mathrm{E}\left[\left(\Delta[\ln (S(t))]-M_{1}^{(j d)}\right)^{4}\right] \\
\sim & 24 \lambda \Delta t\left((1-p) \mu_{2}^{4}+p \mu_{1}^{4}\right) \\
& +24\left(7 p \mu_{1}^{4}-5 p \mu_{2}^{4}+6 \mu_{2}^{4}\right. \\
& +p \mu_{1}\left(\mu_{2}^{3}-p \mu_{1}^{3}\right)-p^{2} \mu_{1} \mu_{2}\left(\mu_{2}^{2}+\mu_{1}^{2}\right) \\
& \left.+p \mu_{2}\left(\mu_{1}^{3}-p \mu_{2}^{3}\right)\right)(\lambda \Delta t)^{2} \\
& +12\left(p \mu_{1}^{2}+(1-p) \mu_{2}^{2}\right)\left(\sigma_{d}^{2} \Delta t\right)(\lambda \Delta t) \\
& +3 \sigma_{d}^{4} \Delta t^{2} .
\end{aligned}
$$

All $\mathrm{O}\left((\lambda \Delta t)^{3}\right)$ and $\mathrm{O}\left((\lambda \Delta t)^{4}\right)$ are omitted in $M_{4}^{(j d)}$. 


\section{F. Skewness and Kurtosis}

In this paper, the skewness and kurtosis are the main benchmarks used to compare the three jump-diffusion models. Therefore, it is important to get $M_{3}^{j d}$ and $M_{4}^{j d}$ in order to get the theoretical skewness and kurtosis coefficient for these three models to sufficient accuracy for a satisfactory comparison.

- Skewness coefficient: $\beta_{3}^{(\mathrm{jd})} \equiv M_{3}^{(\mathrm{jd})} /\left(M_{2}^{(\mathrm{jd})}\right)^{1.5}$.

- Kurtosis coefficient: $\beta_{4}^{(\mathrm{jd})} \equiv M_{4}^{(\mathrm{jd})} /\left(M_{2}^{(\mathrm{jd})}\right)^{2}$.

Sometimes, the kurtosis is represented as the excess kurtosis coefficient by subtracting three from the above kurtosis coefficient definition so that the excess kurtosis coefficient is zero for the normal distribution.

\section{Parameter Estimations}

The basic point of view, here, is that the financial markets are considered to be a moderate size simulation of one of these three jump-diffusion processes.

\section{A. Empirical Data}

We use Standard and Poor's 500 (S\&P500) stock index in the decade 1992-2001 [13] as the sample of the financial market since it is in general viewed as one big mutual fund so that it is less dependent on the peculiar behavior of any one stock.

Let $n^{(\mathrm{sp})}=2522$ be the number of daily closings $S_{s}^{(\mathrm{sp})}$ for $s=1: n^{(\mathrm{sp})}$, such that there are $n s=2521 \log$-returns,

$$
\Delta\left[\ln \left(S_{s}^{(\mathrm{sp})}\right)\right] \equiv \ln \left(S_{s+1}^{(\mathrm{sp})}\right)-\ln \left(S_{s}^{(\mathrm{sp})}\right),
$$

for $s=1: n s \log$-returns, with

- Mean:

$$
M_{1}^{(\mathrm{sp})}=\frac{1}{n s} \sum_{s=1}^{n s} \Delta\left[\ln \left(S_{s}^{(\mathrm{sp})}\right)\right] \simeq 4.015 \mathrm{e}-4 .
$$

- Variance:

$$
\begin{aligned}
M_{2}^{(\mathrm{sp})} & =\frac{1}{n s-1} \sum_{s=1}^{n s}\left(\Delta\left[\ln \left(S_{s}^{(\mathrm{sp})}\right)\right]-M_{1}^{(\mathrm{sp})}\right)^{2} \\
& \simeq 9.874 \mathrm{e}-5
\end{aligned}
$$

- Skewness coefficient:

$$
\beta_{3}^{(\mathrm{sp})} \equiv \frac{M_{3}^{(\mathrm{sp})}}{\left(M_{2}^{(\mathrm{sp})}\right)^{1.5}} \simeq-0.2913<0,
$$

where $\beta_{3}^{(n)}=0$ is the normal distribution value and $M_{3}^{(\mathrm{sp})}$ is the 3 rd central log-return moment of the data.

- Kurtosis coefficient:

$$
\beta_{4}^{(\mathrm{sp})} \equiv \frac{M_{4}^{(\mathrm{sp})}}{\left(M_{2}^{(\mathrm{sp})}\right)^{2}} \simeq 7.804>3,
$$

where $\beta_{4}^{(n)}=3$ is the normal distribution value and $M_{4}^{(\mathrm{sp})}$ is the 4 th central log-return moment of the data.

\section{B. Multinomial Maximum Likelihood Estimation}

In a previous paper [8], the multinomial maximum likelihood estimation of model parameters is justified for binned financial data, but applied to very general binned data. The main idea for this method is the following:

- Step 1: Sample Data is sorted into $n b$ bins and get the sample frequency $f_{b}^{(s p)}$, for $b=1: n b$.

- Step 2: Get the theoretical jump-diffusion frequency with parameter vector $\boldsymbol{x}$ :

$$
f_{b}^{(\mathrm{jd})}(\boldsymbol{x}) \equiv n s \int_{B_{b}} \phi^{(\mathrm{jd})}(\eta ; \boldsymbol{x}) d \eta,
$$

where $B_{b}$ is the $b$ th bin.

- Step 3: Minimize the objective function:

$$
y(\boldsymbol{x}) \equiv-\sum_{b=1}^{n b}\left[f_{b}^{(\mathrm{sp})} \ln \left(f_{b}^{(\mathrm{jd})}(\boldsymbol{x})\right)\right],
$$

which is the negative of the likelihood. Getting the negative of the maximum likelihood corresponds to the minimizing fminsearch function implementation of the Nelder-Mead down-hill simplex direct search method in MATLAB. The Nelder-Mead method [12] is used to get the optimal parameters $\boldsymbol{x}^{*}$ for the three compared models, respectively. The Nelder-Mead is usually faster than other optimization methods when it works. Some comparisons with our multidimensional golden section search method for the financial parameter estimation problem are given in [8].

\section{Jump-Diffusion Moment Estimation Constraints}

For the jump-diffusion model with log-normal and loguniform jump-amplitude, there are five (5) free jumpdiffusion parameters:

$$
\left\{\mu_{l d}, \sigma_{d}^{2}, \mu_{j}, \sigma_{j}^{2}, \lambda\right\} .
$$

For the stock return jump-diffusion model with log-doubleexponential jump-amplitude, there are six (6) free jumpdiffusion parameters:

$$
\left\{\mu_{l d}, \sigma_{d}^{2}, \mu_{1}, \mu_{2}, p, \lambda\right\} .
$$

So, to reduce this set to a reasonable number, the multinomial maximum likelihood estimation is subjected to the mean and variance constraints:

$$
M_{1}^{(\mathrm{sp})}=M_{1}^{(\mathrm{jd})}
$$

and

$$
M_{2}^{(\mathrm{sp})}=M_{2}^{(\mathrm{jd})} .
$$

So, for the log-normal and log-uniform jump-diffusion model, the two diffusion parameters, $\mu_{l d}$ and $\sigma_{d}$, are eliminated by

$$
\mu_{l d}=\left(M_{1}^{(s p)}-\mu_{j} \lambda \Delta t\right) / \Delta t
$$

and

$$
\sigma_{d}^{2}=\left(M_{2}^{(s p)}-\left(\sigma_{j}^{2}(1+\lambda \Delta t)+\mu_{j}^{2}\right) \lambda \Delta t\right) / \Delta t,
$$


the latter is subject to positivity constraints, for fixed and small $\Delta t \ll 1$. Hence, only three free parameters are left:

$$
\boldsymbol{x}=\left\{\mu_{j}, \sigma_{j}^{2}, \lambda\right\} .
$$

For the log-double-exponential jump-diffusion model, two parameters $\mu_{l d}$ and $\sigma_{d}$ are eliminated by

$$
\mu_{l d}=\left(M_{1}^{(s p)}-\left(-p \mu_{1}+(1-p) \mu_{2}\right) \lambda \Delta t\right) / \Delta t
$$

and

$$
\begin{aligned}
\sigma_{d}^{2}= & \left(M_{2}^{(s p)}-2\left(p\left(\mu_{1}^{2}-\mu_{2}^{2}\right)+\mu_{2}^{2}\right) \lambda \Delta t\right. \\
& +\left(p(2-p) \mu_{1}^{2}+2 p(1-p) \mu_{1} \mu_{2}\right. \\
& \left.\left.+\left(1-p^{2}\right) \mu_{2}^{2}\right)(\lambda \Delta t)^{2}\right) / \Delta t
\end{aligned}
$$

Then, four (4) free parameters are left:

$$
\boldsymbol{x}=\left\{\mu_{1}, \mu_{2}, p, \lambda\right\},
$$

with significantly more computational cost.

\section{Numerical Results, Figures and Discussion}

The multinomial maximum likelihood estimation given here is used to estimate the jump-diffusion parameters. The numerical optimization was performed using the MATLAB 6.5 [11] computing system's fminsearch function, an implementation of the down-hill simplex direct search method of Nelder and Mead [12].

For the log-normal and log-uniform model, the same starting point $\boldsymbol{x}_{0}$ is used. For the log-double-exponential model, the different starting point $\boldsymbol{x}_{0}$ is used: $\mu_{1}$ and $\mu_{2}$ are from the estimation of the $\mu_{j}$ of the log-uniform model, $p \simeq 0.6>0.5$ means more likely downward jumpamplitudes and the $\lambda \Delta t$ value are the same as the log-normal and log-uniform.

The empirical data used in the estimation are the S\&P500 daily closing log-returns from the decade 1992-2001. In Figure 1 is the histogram of bin frequencies using 100 centered bins. Note the long, relatively thick tails signifying crashes in the negative tails and rallies in the positive tails, where the normal distribution or the double-exponential would have insignificant tail values. The ragged appearance of the histogram resembles the random simulation of a density using a moderate, but inadequate, sample size. The rare, larger jump events are difficult to see in the scale of the figure.

However, if the histogram frequencies are multiplied by the centered value of the bin log-return, then the larger jumps are clearly visible. This moment-histogram is called a hysteriagram since it magnifies the larger jumps and corresponds to the extreme behavioral reaction of some investors. The hysteriagram for the S\&P500 is given in Figure 2 and clearly indicates the inadequacy of using a log-normal and the log-double-exponential to characterize significant large events.

Figure 3 shows that the log-normal jump-amplitude model hysteriagram exhibits too thin tails that decay too fast with the jump magnitude. From (II.4) it can be seen that the bin

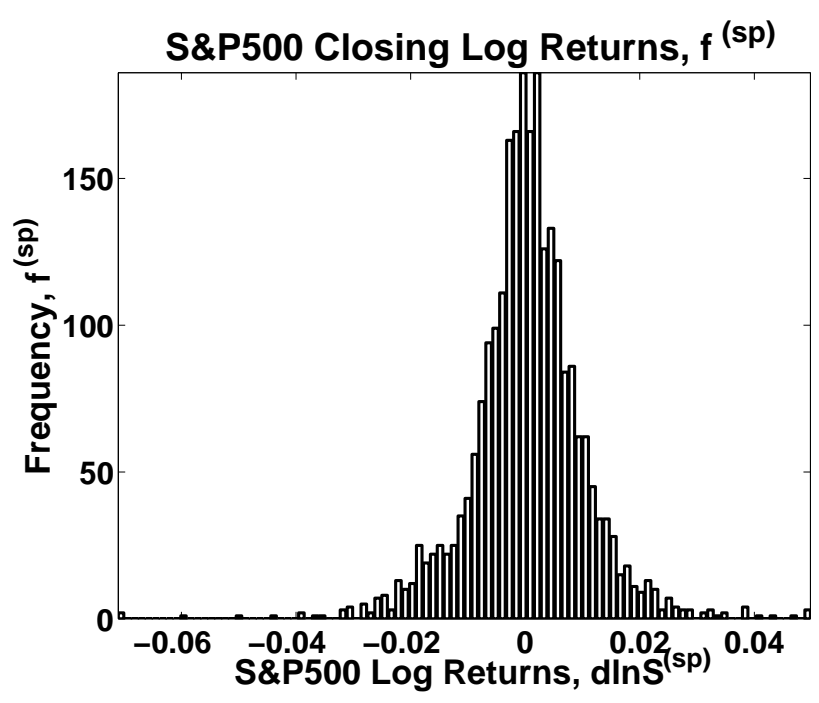

Fig. 1. Histogram of S\&P500 log-return frequencies for the decade 19922001, using 100 bins.

\section{S\&P500 Hysteriagram: X*Frequency}

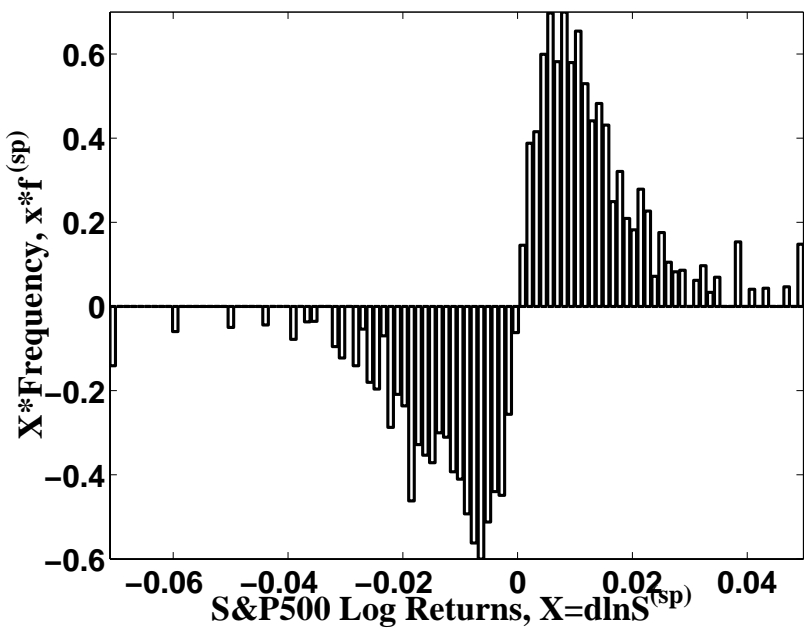

Fig. 2. Hysteriagram of S\&P500 log-return frequencies multiplied by the average bin log-return value for the decade 1992-2001, using 100 bins.

distribution for sufficiently narrow bins will be a Poisson sum of normal distributions, so will have thin exponential Gaussian tails. The corresponding histogram for the lognormal, not shown here, does not show enough visual detail to sufficiently distinguish it from the other jump-amplitude models.

Figure 4 shows that the log-uniform jump-amplitude model hysteriagram exhibits much thicker tails that decay more slowly with the jump magnitude, but do not capture the largest negative jump in Figure 2. The secant-normal densities in (II.6) help counter the normal distribution tendency to having exponential thin tails, but not for beyond the largest jump values of the log-returns.

Figure 5 shows that the log-double-exponential jumpamplitude model hysteriagram exhibits too thin tails that decay too fast with the jump magnitude that is very similar 


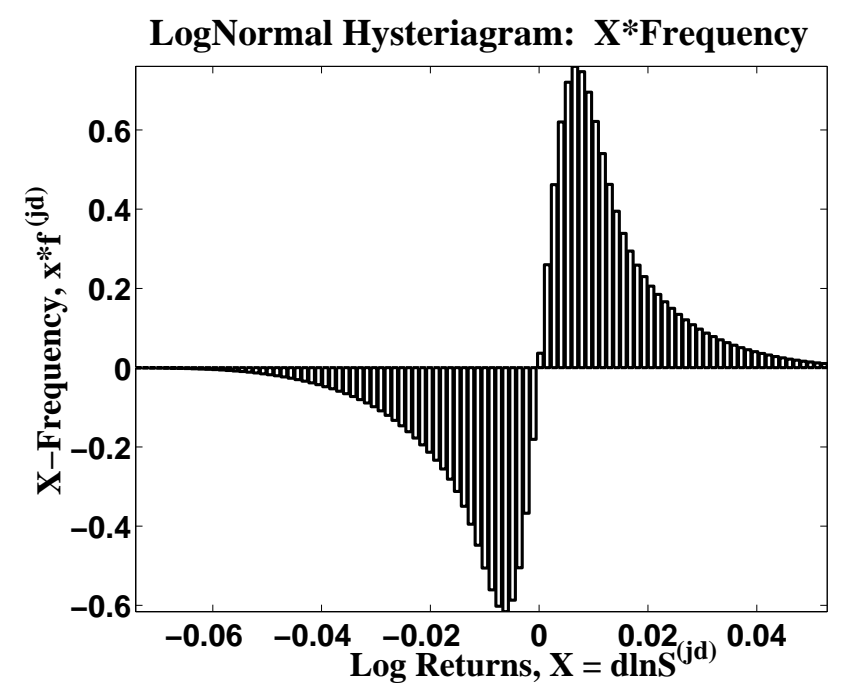

Fig. 3. Hysteriagram of the predicted log-returns frequencies multiplied by the average bin log-return value for the log-normal jump-amplitude jump-diffusion model, using 100 bins.

\section{LogUniform Hysteriagram: $X^{*}$ Frequency}

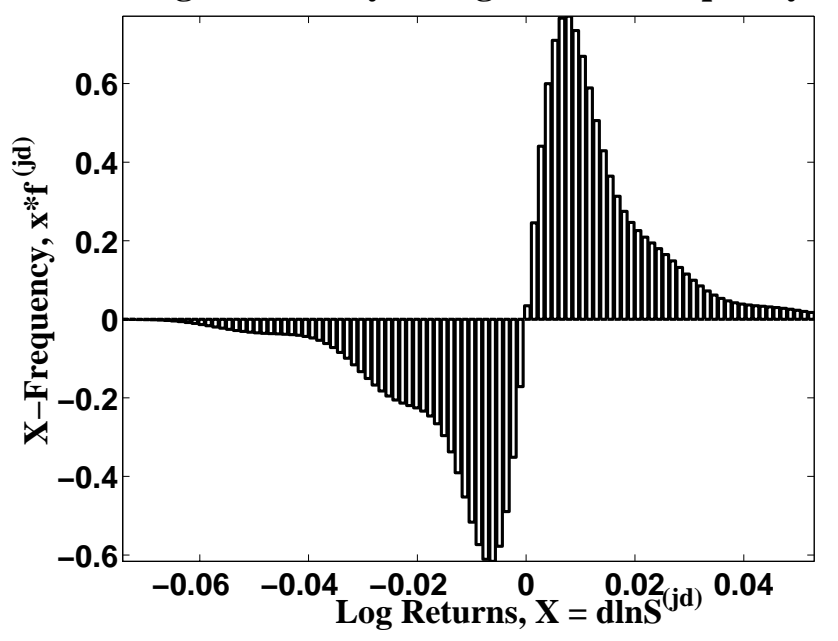

Fig. 4. Hysteriagram of the predicted log-returns frequencies multiplied by the average bin log-return value for the log-uniform jump-amplitude jump-diffusion model, using 100 bins.

to the log-normal jump-amplitude model. The convolution of normal and exponential distributions in (II.8), like the normal jump-amplitude model, can only lead to exponential thin tails.

Hence, the log-uniform model is a qualitatively better model for the S\&P500 data, since the tails are thick enough to generate more of the larger jumps seen in the S\&P500 data in Fig. 2 than the other two distributions.

From Table I, we can have a quantitative estimate of the derived distribution parameters $\mu_{d}, \sigma_{d}, \mu_{j}, \sigma_{j}, \lambda$. Since the trading days per year are about 250 days, it is not likely that the jumps rate is more than 100 per year because the finance market should be kept stable. So, $\lambda \simeq 59$ for the log-uniform is more reasonable, considering that the uniform

\section{LogDblExp Hysteriagram: X*Frequency}

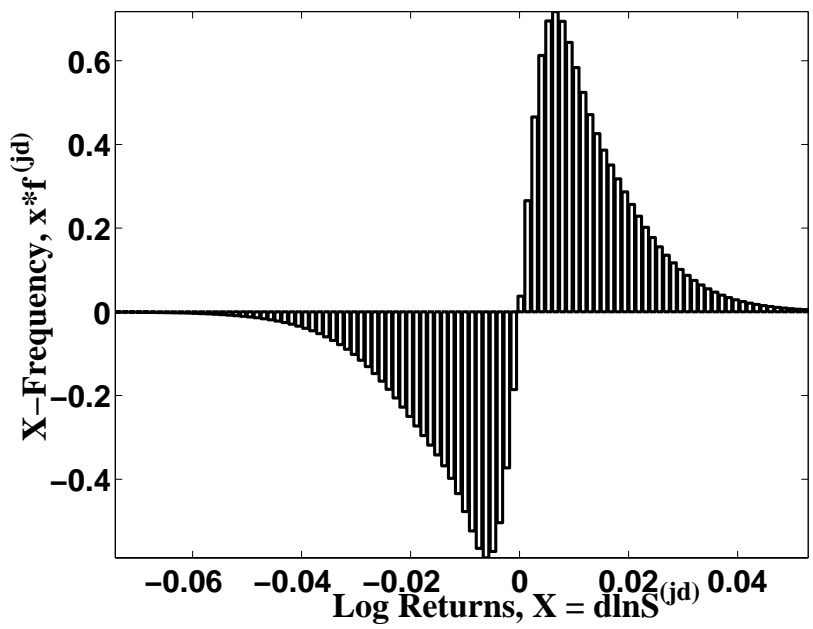

Fig. 5. Hysteriagram of the predicted log-returns frequencies multiplied by the average bin log-return value for the log-double-exponential jumpamplitude jump-diffusion model, using 100 bins.

jump distribution spans the crash to the rally data. The near-zero peaks of the normal and double-exponential lead to more than double the uniform jump rate. Note that the jump rate includes all size jumps, including those hidden by the log-normal part of the log-return distribution. In the table the overall jump mean $\mu_{j}$ is given for the purpose of comparison, but for the double-exponential, the negative jump mean is $\mu_{j, 1}=-\mu_{1}=-3.63 \mathrm{e}-3$ and the positive jump mean is $\mu_{j, 2}=\mu_{2}=+3.24 \mathrm{e}-3$. For the double-exponential, the probability of negative jumps is $p=0.481$ and that for positive jumps is $(1-p)=0.519$. For the other parameters, we can use the most-common value among these three models. Then, we get the other parameter estimations for the log-uniform as the following: $\mu_{d} \simeq 0.20, \sigma_{d} \simeq 0.085, \mu_{j} \simeq$ $-1.6 \mathrm{e}-3$ and $\sigma_{j} \simeq 0.015$. Hence, overall the log-uniform has a better estimation for these derived parameters.

TABLE I

Comparison summary of derived distribution parameters for the log-normal, log-uniform and log-double-exponential jump-diffusion models, respectively.

\begin{tabular}{|c||c|c|c|c|c|}
\hline Model & $\mu_{d}$ & $\sigma_{d}$ & $\mu_{j}$ & $\sigma_{j}$ & $\lambda$ \\
\hline \hline Normal & 0.199 & 0.0907 & $-7.28 \mathrm{e}-4$ & $9.25 \mathrm{e}-3$ & 128. \\
\hline Uniform & 0.198 & 0.0865 & $-1.63 \mathrm{e}-3$ & $1.53 \mathrm{e}-2$ & 59.4 \\
\hline Dbl-Exp & 0.125 & 0.0791 & $-6.15 \mathrm{e}-5$ & $4.86 \mathrm{e}-3$ & 338. \\
\hline
\end{tabular}

From Table II, the difference of skewness and kurtosis between the estimate value and the observed are $16 \%$ and $-2.2 \%$ for the log-uniform model. These results are better when considering both coefficients than the other two models' results, except the difference skew for the double-exponential is the lowest, though the difference in the kurtosis is highest for the double-exponential. Also, given is the terminating multinomial maximum likelihood using the negative of minimum of the objective in (III.2), essentially 
the same for all models with the same stopping criterion being used.

TABLE II

The skewness and kurtosis coefficients for the three models are compared to S\&P500 values, respectively, and Multinomial Maximum Likelihood $(\mathrm{MML} \simeq-\min [y(\mathbf{x})])$.

\begin{tabular}{|c||c|c|c|c|c|}
\hline Model & $\beta_{3}$ & $\%$ & $\beta_{4}$ & $\%$ & MML \\
\hline \hline Normal & -0.196 & -32.7 & 8.90 & 14.0 & $1.118 \mathrm{e} 4$ \\
\hline Uniform & -0.387 & +16.3 & 7.63 & -2.21 & $1.117 \mathrm{e} 4$ \\
\hline Dbl-Exp & -0.279 & -4.28 & 12.2 & 57.0 & $1.119 \mathrm{e} 4$ \\
\hline \hline S\&P500 & -0.291 & 0.0 & 7.80 & 0.0 & - \\
\hline
\end{tabular}

From the Table III, we can see that the log-normal and log-uniform models take the same order of magnitude of iterations and function evaluation, the log-normal model parameter estimate takes $1 / 7$ of the time to execute. One reason is that the log-normal requires only one normal distribution calculation for each jump $k$ in (II.4), while the others required the calculation of either an integral of the secant-normal or of several normal distributions. However, the extra parameter needed for the double-exponential means the iteration count, the function evaluation count and the timings will be much greater for the double-exponential. The computational efforts for the uniform and doubleexponential models were reduced by using integration by parts to reduce the original double bin distribution integrals to single integrals. An added advantage of such a reduction also can improve accuracy and speed of computation. The reduced formulas are too lengthy to report here. The reduction of the original double-exponential bin integrals to single integrals led to exponential catastrophic cancellation problems, in that exponential factors of that model interfered with the absolute error threshold of the MATLAB integration function quadl for the most negative bin locations causing small violations of positive probability properties. Absorption of these exponential factors into the integrands accurately corrected the error threshold problem.

TABLE III

Comparison summary of computational performance measures:

\begin{tabular}{|c||c|c|c|c|}
\hline $\begin{array}{c}\text { Model } \\
\text { Used }\end{array}$ & $\begin{array}{c}\text { Number } \\
\text { Parms. }\end{array}$ & $\begin{array}{c}\text { Number } \\
\text { Iters. }\end{array}$ & $\begin{array}{c}\text { Function } \\
\text { Evals. }\end{array}$ & $\begin{array}{c}\text { Timings } \\
(\mathrm{sec})\end{array}$ \\
\hline \hline Normal & 3 & 69 & 129 & 28.7 \\
\hline Uniform & 3 & 54 & 101 & 211 \\
\hline Dbl-Exp & 4 & $200^{*}$ & 342 & 2638 \\
\hline
\end{tabular}

Combined Legend for Table I, Table II and Table III:

- Normal: Log-normal jump-amplitude.

- Uniform: Log-uniform jump-amplitude.

- Dbl-Exp: Log-double-exponential jump-amplitude.

- Maximum Number of Iterations: $200^{*}$.

- Using same tolerances: tolx $=5 \mathrm{e}-6$ and toly $=5 \mathrm{e}-6$.

- Using P4@1.6GHz CPU computer processor with MATLAB.

\section{SUMMARY AND CONCLUSION}

From the above theoretical and data analysis, we can get the following conclusions:

- The log-uniform model is the best overall among the three models, qualitatively in terms of genuinely representing the fat tail property of real-world market distributions and quantitatively in terms of reasonable overall higher moments, i.e., both skewness and kurtosis.

- The log-normal model runs faster than the other two models. The reason is that the optimization algorithm needs only single bin integrals over a normal density for the log-normal model. On the other hand, the integration by parts technique can be used to reduce the computational effort for the log-uniform and logdouble-exponential models. However, the deficiencies of the log-normal model demonstrates that the distribution that is better analytically is not necessarily a better model for financial markets, i.e., finding a better model may be counter to the desire to obtained closed form solutions.

- The results for the log-normal and log-doubleexponential jump amplitude models are qualitatively similar. Both of them have exponentially small tails and peaks in the center making small jumps more likely. If there are small jumps, they are not much different from diffusion fluctuations since the diffusion part of the jump-diffusion model dominates the stock log-Return process (II.2) in this case. If there are large jumps, then the exponentially small tails of their distributions can not contribute too much to the flat and thick tails of the real world financial markets. Therefore, these two models has some intrinsic defects and are not not recommended for monitoring the dynamics of the finance markets.

- For the future research and considerations.

1) To develop better way to the fit rare, jump events.

2) To improve the log-uniform model, the stochastic volatility should be considered since in the real world the implied volatility curve is not a constant, but 'smile' curve.

3) To consider the option price problems based on the log-uniform model and try to get the exact or approximate solutions to these problems if it is possible. Now the option market grows very fast, we must face these problems and put the model under the real-world finance markets' test.

\section{References}

[1] T. G. Andersen, L. Benzoni, and J. Lund, An Empirical Investigation of Continuous-Time Equity Return Models, J. Finance, vol. 57(3), 2002, pp. 1239-1284.

[2] F. Black and M. Scholes, The Pricing of Options and Corporate Liabilities, J. Political Economy, vol. 81, 1973, pp. 637-659.

[3] F. B. Hanson and J. J. Westman, "Optimal Consumption and Portfolio Control for Jump-Diffusion Stock Process with Log-Normal Jumps," Proc. 2002 Amer. Control Conf., 2002, pp. 4256-4261. 
[4] F. B. Hanson and J. J. Westman, "Jump-Diffusion Stock Return Models in Finance: Stochastic Process Density with Uniform-Jump Amplitude," Proc. 15th Int. Sympos. Mathematical Theory of Networks and Systems, 2002, pp. 1-7.

[5] F. B. Hanson and J. J. Westman, "Portfolio Optimization with JumpDiffusions: Estimation of Time-Dependent Parameters and Application," Proc. 2002 Conf. Decision and Control, 2002, pp. 377-382.

[6] F. B. Hanson and J. J. Westman, "Jump-Diffusion Stock-Return Model with Weighted Fitting of Time-Dependent Parameters," Proc 2003 Amer. Control Conf., 2003, pp. 4869-4874.

[7] F. B. Hanson and J. J. Westman, Applied Stochastic Processes and Control for Jump-Diffusions: Modeling, Analysis and Computation SIAM Books, Philadelphia, PA, to appear 2004-2005.
[8] F. B. Hanson, J. J. Westman and Z. Zhu, Multinomial Maximum Likelihood Estimation of Market Parameters for Stock Jump-Diffusion Models, Contemporary Mathematics, to appear Spring 2004.

[9] S. G. Kou, A Jump Diffusion Model for Option Pricing, Management Science, vol. 48, 2002, pp. 1086-1101.

[10] R. C. Merton, Option Pricing when Underlying Stock Returns are Discontinuous, J. Financial Economics, vol. 3, 1976, pp. 125-144.

[11] C. Moler et al., Using MATLAB, Version 6, Mathworks, Natick, MA, 2000.

[12] J. A. Nelder and R. Mead, A Simplex Method for Function Minimization, Computer Journal, vol, 7, 1965, pp. 308-313.

[13] Yahoo! Finance, Historical Quotes, S \& P 500 Symbol - SPC, URL: http: //chart.yahoo.com/. February 2002 